\title{
Discriminating Features for Writer Identification
}

\author{
Zachary A. Daniels \\ Computer Science \& Engineering Dept. \\ Lehigh University \\ 19 Memorial Dr West, Bethlehem, PA 18017 USA \\ Email: zad309@lehigh.edu
}

\author{
Henry S. Baird \\ Computer Science \& Engineering Dept. \\ Lehigh University \\ 19 Memorial Dr West, Bethlehem, PA 18017 USA \\ Email: baird@cse.lehigh.edu
}

\begin{abstract}
This paper investigates highly discriminating features for writer identification for off-line handwritten text lines and passages. Five categories of features are tested: slant and slant energy, skew, pixel distribution, curvature, and entropy. Three experiments are run utilizing the IAM Handwriting Database and the ICDAR 2011 Writer Identification Contest dataset: the first, on 10 writers from the IAM dataset; the second, on 50 writers from the IAM dataset; and the third, strictly following the methodology of the 2011 ICDAR Writer Identification Contest. When compared to the other methodologies tested in the ICDAR competition, ours ranked fourth out of nine. These features support high recognition rates and are competitive with other state of the art methods for writer identification.
\end{abstract}

\section{INTRODUCTION}

The problem of writer identification addresses whether or not a classifier can be created that is able to identify the writer of some handwritten text with a high accuracy given a set of handwritten documents from many writers. Writer identification is a well-studied problem [5] [4] [14] [19] [9] [16] [11]. Sreeaj and Sumam provide an extensive survey of the state of the art in writer identification [18].

Writer identification is an area of great interest and importance. It is especially important for verification purposes. For example, validating signatures on checks and legal documents such as wills are two such applications of writer identification as a means of validation. Likewise, writer identification of documents is extremely important in legal matters where handwritten documents are often used as evidence.

We have conducted experiments using two publicly available datasets: the IAM Handwriting Database [13] and ICDAR 2011 Writer Identification Contest dataset [12]. Experiments run on the IAM Handwriting Database use off-line, individual handwritten lines of English language text for training and testing; the text line images are greyscale. The experiments run on the ICDAR 2011 Writer Identification Contest dataset use offline, handwritten short passages of natural language text roughly one to two paragraphs long contained in single binary images; the text passages are written in four languages. We experimented on isolated text lines and entire paragraphs but not on isolated words nor signatures.

Five categories of text-independent features were explored: slant and slant energy, skew, pixel distribution, curvature, and entropy. With the exception of the pixel distribution feature, features operate on text lines and paragraphs. The pixel distribution feature only operates on the text line level. The paper is organized as follows: in the next section, individual features are introduced. In Section 3, the experiments used to test the quality of the introduced features are described. Section 4 discusses the results of the experiments and introduces future work.

\section{Selected Features}

\section{A. Slant and Slant Energy}

Slant is the deviation in the direction of handwriting from the vertical. In order to begin calculating slant, the Sobel operator is applied to the greyscale image of a handwriting sample in order to detect edges [15]. Two kernels are convolved across the greyscale image. The following kernels are convolved across the image horizontally and vertically:

$$
\mathbf{K}_{\mathbf{x}}=\left(\begin{array}{ccc}
-1 & 0 & 1 \\
-2 & 0 & 2 \\
-1 & 0 & 1
\end{array}\right), \mathbf{K}_{\mathbf{y}}=\left(\begin{array}{ccc}
-1 & -2 & -1 \\
0 & 0 & 0 \\
1 & 2 & 1
\end{array}\right)
$$

The purpose of the Sobel operator is to approximate the gradient of an image at each pixel. When the kernels $\mathbf{K}_{\mathbf{x}}$ and $\mathbf{K}_{\mathbf{y}}$ are convolved over the greyscale representation of the image $\mathbf{I}$, the resulting matrices $\mathbf{G}_{\mathbf{x}}$ and $\mathbf{G}_{\mathbf{y}}$ represent the horizontal and vertical derivative approximations, respectively. To get the magnitude of the gradient of the image $\mathbf{G}$ at each point, the following formula is applied:

$$
\mathbf{G}=\sqrt{\mathbf{G}_{\mathbf{x}}{ }^{2}+\mathbf{G}_{\mathbf{y}}{ }^{2}}
$$

Slant at each pixel of the image $S$ can then be calculated as:

$$
\mathbf{S}=\arctan \frac{\mathbf{G}_{\mathbf{y}}}{\mathbf{G}_{\mathbf{x}}}
$$

Four features relating directly to slant are extracted: the approximate dominant slant, the approximate dominant slant in the positive direction, the approximate dominant slant in the negative direction, and the ratio of instances where the gradient is positive in the image to the total number of instances where gradient can be calculated. To calculate the average dominant slant, let

$$
\begin{aligned}
\mathbf{G} & =\left(\begin{array}{cccc}
g_{1,1} & g_{1,2} & \cdots & g_{1, n} \\
g_{2,1} & g_{2,2} & \cdots & g_{2, n} \\
\vdots & \vdots & \ddots & \vdots \\
g_{m, 1} & g_{m, 2} & \cdots & g_{m, n}
\end{array}\right) \\
\mathbf{S} & =\left(\begin{array}{cccc}
s_{1,1} & s_{1,2} & \cdots & s_{1, n} \\
s_{2,1} & s_{2,2} & \cdots & s_{2, n} \\
\vdots & \vdots & \ddots & \vdots \\
s_{m, 1} & s_{m, 2} & \cdots & s_{m, n}
\end{array}\right)
\end{aligned}
$$




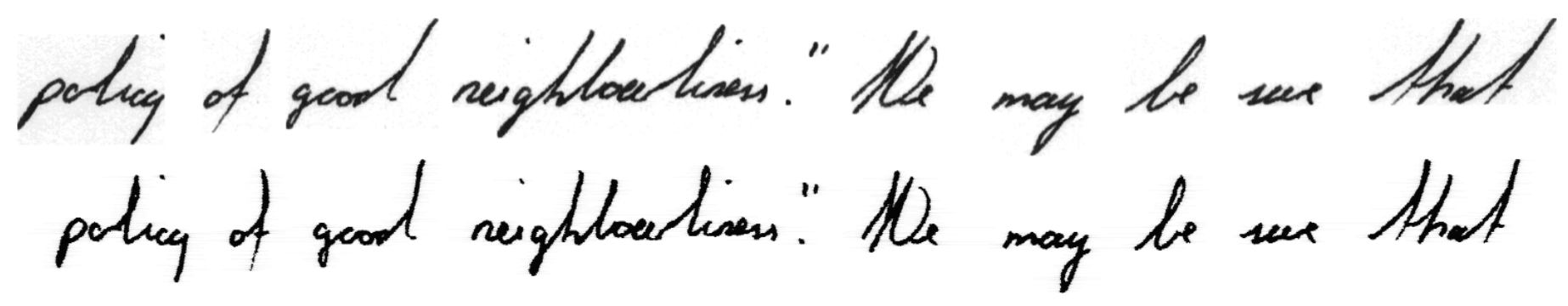

Fig. 1. Top: Line of handwritten text before slant-correction, Bottom: Line of handwritten text after slant-correction

Then,

$$
\bar{S}=\frac{\sum_{m} \sum_{n} s_{m, n} * g_{m, n}}{\sum_{m} \sum_{n} g_{m, n}}
$$

For the positive direction calculation, the same procedure is followed, but every negative angle is changed to a value of zero. For the negative direction calculation, each positive angle is changed to a value of zero.

Slant energy is also used as a feature. The image is binarized. The text sample is slant-corrected by shearing at the dominant slant, an example of which can be seen in Fig. 1. The projection profile in the vertical direction is calculated, and each value is squared in order to calculate the slant energy at a particular $\mathrm{x}$-coordinate in the image. The peaks and valleys of the energy profile are located and summed separately, and the sum of the valleys is subtracted from the sum of the peaks (which helps to smooth jagged peaks while maintaining the relevant information contained in the energy profile). The resulting number is called as the 'slant energy' feature.

\section{B. Skew}

Skew is the measure of how much a text line differs from being parallel with the horizontal. In order to calculate skew, a document or line is rotated by increments of 0.05 degrees. For each rotation, the energy of the horizontal projection profile is calculated using a method similar to that presented in the discussion on slant. The skew correlates to the angle of rotation where the energy is maximized [3]. Skew is used as a feature, and skew-correction is used as a preparatory measure when calculating feature values yet to be introduced. Fig. 2 shows an example text line before and after it is corrected for skew with the lower baseline used as a reference point.

\section{Pixel Distribution}

The text image is binarized and corrected for skew. The horizontal projection profile is calculated. The location of the largest peak of the profile is determined. This peak approximates the centerline of a text line. Fig. 3 shows the projection profile with a line denoting its approximate centerline. The number of pixels above and below centerline are counted. In the figure, area $A$ to the left of the centerline represents the number of pixels above the centerline, and the area to the right of the centerline, $B$, represents the pixels below the centerline. The ratio between these two numbers is calculated and used as a feature.

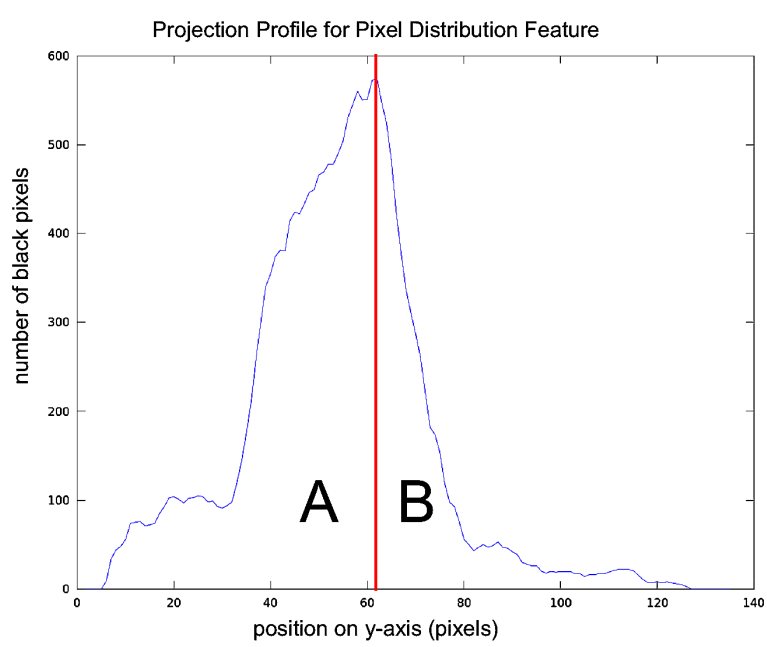

Fig. 3. Profile of a single text line image projected horizontally onto a vertical line. The red line denotes the centerline.

\section{Curvature}

Curvature is the degree to which a line deviates from being straight. Several features relating to curvature are investigated. To begin, the text image must be binarized. The boundaries of the connected components of the image are calculated using an 8-connectivity labeling algorithm. The radius of curvature for every set of 3 points each separated by 5 boundary points (typically edge pixels) is calculated. The average radius of curvature is used as a feature.

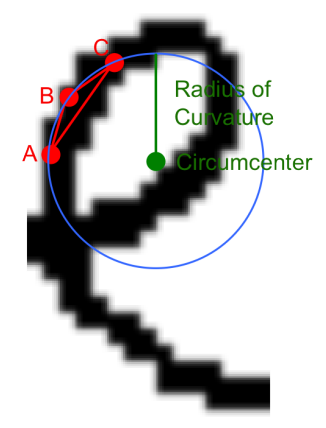

Fig. 4. Calculating the radius of curvature 


\section{Moxket, even if it meant ditionar on Moxket, even if it meant an uncon ditional surrender.}

Fig. 2. Top: Line of handwritten text before skew-correction, Bottom: Line of handwritten text after skew-correction

Please refer to Fig. 4 for a visual representation of how three boundary points can be used to calculate the radius of curvature at some point in an image. Use the three boundary points to form a triangle. Find the circumscribed circle of the triangle. Then the radius of the circle can be calculated using Eq. (1) [7]. The average radius of curvature is then easily calculated.

Eight more features can be easily obtained by bucketing the direction of curvature whenever radius of curvature is calculated. The direction of curvature is separated into eight buckets $[+\mathrm{x},+\mathrm{y},-\mathrm{x},-\mathrm{y},(+\mathrm{x},+\mathrm{y}),(+\mathrm{x},-\mathrm{y}),(-\mathrm{x},+\mathrm{y})$, and $(-\mathrm{x},-\mathrm{y})]$ based on the $\mathrm{x}$ and $\mathrm{y}$ components of the boundary points used to calculate the radius of curvature, and the proportions of each direction are used as features.

\section{E. Entropy}

Entropy is a measure of the randomness of an image. Entropy is calculated as follows:

$$
E=-\sum \mathbf{P} \cdot \log _{2} \mathbf{P}
$$

where $\mathbf{P}$ is a vector containing the probabilities of each pixel value ( 0 or 1 for binary and 0 to 255 for greyscale) in the image [8].

\section{EXPERIMENTS}

Experiments were conducted over two datasets: the IAM Handwriting Database and ICDAR 2011 Writer Identification Contest dataset.

A preliminary experiment was run on handwriting samples from ten writers in order to quickly test the feature extraction and classification algorithms. Two scientifically rigorous experiments were then run: the first on 50 writers from the IAM dataset and the second following the methodology of the 2011 ICDAR Writer Identification Contest.

Experiment 1 Ten writers were selected from the IAM Database. For each writer, five lines from five documents (for a total of 25 instances) were used for training. All remaining lines from the documents were used for testing. After feature values were extracted from the training set, a whitening transformation was applied to the data. A nearest neighbor classifier was used with the means of the feature values of the training instances for each class being used instead of the feature values of the individual training instances. This was done to reduce noise. Euclidean distance was used for calculating similarity. The error rate was $10.7 \%$. When 6-nearest neighbor was used on the original, whitened training instances (i.e. not the means), a $9.3 \%$ error rate was achieved.
TABLE I. "SOFT" RESULTS ON 2011 ICDAR WRITER IDENTIFICATION CONTEST DATASET: AN ERROR IS SAID TO HAVE OCCURRED WHEN A DOCUMENT BY THE SAME WRITER DOES NOT APPEAR IN THE $n$ MOST SIMILAR DOCUMENTS

\begin{tabular}{|l|l|l|l|l|}
\hline Language & Top-1 & Top-2 & Top-5 & Top-10 \\
\hline Entire Dataset & 97.1 & 99.5 & 100 & 100 \\
Greek & 90.4 & 94.2 & 100 & 100 \\
English & 94.2 & 98.1 & 100 & 100 \\
French & 94.2 & 98.1 & 100 & 100 \\
German & 100 & 100 & 100 & 100 \\
\hline
\end{tabular}

Experiment 2 Fifty writers were selected from the IAM Database. For each writer, five lines from a single document (five instances) were used for training. Ten lines from two different documents for each writer were used for testing. The data was whitened. A nearest neighbor classifier was used with the means of the feature values of the training instances for each class being used instead of the feature values of the individual training instances. Like in the previous experiment, this was done to reduce noise. Euclidean distance was used to calculate similarity. The error rate was $18.4 \%$. Using 6-nearest neighbor on the original, whitened training instances resulted in an error rate of $14.8 \%$.

Experiment 3 The experiment was run using the exact test methods, dataset, and testing software of the 2011 ICDAR Writer Identification Contest for entire text documents. 26 writers copied eight pages of text in four languages. For each pair of document images, a distance value is calculated. Accuracy of the various methodologies are measured using the soft TOP-N and hard TOP-N criterion. It should be noted that the distance calculation algorithm was trained on an experimental set of document images and tested on the full document set previously described. Competing teams utilized edge-based directional probability distribution features, grapheme features, grid microstructure features, K-adjacent segment (KAS) features, features based on the probability distribution of black and white run-lengths, and features that capture orientation and curvature information in writing at different levels of observation. Methodologies tested in the competition were based on the works presented in [2] [1] [20] [10] [6] and [17]. For details about the benchmark dataset, evaluation methods, and results of the competition see [12]. Note that the features presented in this paper were only tested on the first scenario presented in the competition paper, and since the competition required input to be full documents as opposed to individual text lines, the pixel distribution feature was not used for this experiment. Euclidean distance was selected as the distance metric. Results of our method are shown in Tables I, II, and III. We ranked fourth out of the nine systems tested. 


$$
r=\frac{\overline{A B} * \overline{B C} * \overline{C A}}{\sqrt{(\overline{A B}+\overline{B C}+\overline{C A})^{2} *(-\overline{A B}+\overline{B C}+\overline{C A})^{2} *(\overline{A B}-\overline{B C}+\overline{C A})^{2} *(\overline{A B}+\overline{B C}-\overline{C A})^{2}}}
$$

TABLE III. METHOD COMPARED TO COMPETITORS OF 2011 ICDAR WRITER IDENTIFICATION CONTEST ON FULL DOCUMENTS

\begin{tabular}{|l|l|l|l|l|l|l|l|l|}
\hline Method & $I$ & $I I$ & $I I I$ & $I V$ & $V$ & VI & Scenario & Rank \\
\hline Tsinghua & 5 & 5 & 8 & 9 & 7 & 4 & 38 & 1 \\
CS-UMD & 7 & 15 & 8 & 5 & 4 & 4 & 43 & 2 \\
MCS-NUST & 8 & 9 & 10 & 9 & 4 & 4 & 44 & 3 \\
Lehigh & $\mathbf{9}$ & $\mathbf{1 4}$ & $\mathbf{8}$ & $\mathbf{7}$ & $\mathbf{8}$ & $\mathbf{4}$ & $\mathbf{5 0}$ & $\mathbf{4}$ \\
QUBA-b & 11 & 12 & 15 & 4 & 6 & 4 & 52 & 5 \\
Tebessa & 6 & 4 & 18 & 9 & 11 & 6 & 54 & 6 \\
GWU & 17 & 20 & 20 & 13 & 8 & 9 & 87 & 7 \\
QUBA-a & 19 & 22 & 17 & 17 & 10 & 11 & 96 & 8 \\
ECNU & 23 & 25 & 25 & 20 & 16 & 15 & 124 & 9 \\
\hline
\end{tabular}

TABLE II. "HARD” RESULTS ON 2011 ICDAR WRITER IDENTIFICATION CONTEST DATASET: AN ERROR IS SAID TO HAVE OCCURRED WHEN A DOCUMENT BY A DIFFERENT WRITER APPEARS IN THE $n$ MOST SIMILAR DOCUMENTS

\begin{tabular}{|l|l|l|l|}
\hline Language & Top-2 & Top-5 & Top-7 \\
\hline Entire Dataset & 92.8 & 69.2 & 31.3 \\
\hline
\end{tabular}

\section{Discussion, CONClusions, AND Future Work}

The problem of writer identification addresses whether or not a classifier can be created that is able to identify the writer of some handwritten text with a high accuracy given a set of handwritten documents from many writers. This paper addressed the problem of writer identification for off-line handwritten text lines and passages. Five categories of textindependent features were examined: slant and slant energy, skew, pixel distribution, curvature, and entropy. These features resulted in high recognition rates competitive with other state of the art methods for writer identification. Our experience suggests that no single features is dominant; instead, these features work very well in combination.

This research can be expanded in several ways. The same experiments could usefully be run on a greater number of writers from the IAM dataset. The amount of training data used could be varied (e.g. what if the classifier was trained on a single text line instead of five lines?). The ICDAR Writer Identification Competition used two scenarios for the amount of information available. The experiment presented in this paper examined the first scenario where an entire text document was given. It did not examine the second scenario where only images of two text lines were used. Lastly, the only classifier examined in this paper was Nearest Neighbor (both 1-NN and 6-NN). Other classifiers such as SVMs and neural networks should certainly be tried.

\section{REFERENCES}

[1] S. Al-Ma'adeed, A.A. Al-Kurbi, A. Al-Muslih, R. Al-Qahtani, and H. Al Kubisi. Writer identification of arabic handwriting documents using grapheme features. In Computer Systems and Applications, 2008. AICCSA 2008. IEEE/ACS International Conference on, pages 923-924. IEEE, 2008.

[2] S. Al-Ma'adeed, E. Mohammed, and D. Al Kassis. Writer identification using edge-based directional probability distribution features for arabic words. In Computer Systems and Applications, 2008. AICCSA 2008. IEEE/ACS International Conference on, pages 582-590. IEEE, 2008.

[3] Henry S. Baird. Document image analysis. chapter The skew angle of printed documents, pages 204-208. IEEE Computer Society Press, Los Alamitos, CA, USA, 1995.
[4] M. Bulacu and L. Schomaker. Text-independent writer identification and verification using textural and allographic features. Pattern Analysis and Machine Intelligence, IEEE Transactions on, 29(4):701-717, 2007.

[5] M. Bulacu, L. Schomaker, L. Vuurpijl, et al. Writer identification using edge-based directional features. writer, 1:1, 2003.

[6] D. Chawki and S.M. Labiba. A texture based approach for arabic writer identification and verification. In Machine and Web Intelligence (ICMWI), 2010 International Conference on, pages 115-120. IEEE, 2010.

[7] H. Dorrie. 100 Great problems of elementary mathematics. Dover Publications, 1965.

[8] RC Gonsales, RE Woods, and SL Eddins. Digital image processing using MATLAB, 2004.

[9] C. Hertel and H. Bunke. A set of novel features for writer identification. In Audio-and Video-Based Biometric Person Authentication, pages 1058-1058. Springer, 2003.

[10] R. Jain and D. Doermann. Offline writer identification using k-adjacent segments. In Document Analysis and Recognition (ICDAR), 2011 International Conference on, pages 769-773. IEEE, 2011.

[11] G. Leedham and S. Chachra. Writer identification using innovative binarised features of handwritten numerals. In Document Analysis and Recognition, 2003. Proceedings. Seventh International Conference on, pages 413-416. IEEE, 2003.

[12] G. Louloudis, N. Stamatopoulos, and B. Gatos. Icdar 2011 writer identification contest. In Document Analysis and Recognition (ICDAR), 2011 International Conference on, pages 1475-1479. IEEE, 2011.

[13] U.V. Marti and H. Bunke. The IAM-database: an english sentence database for offline handwriting recognition. International Journal on Document Analysis and Recognition, 5(1):39-46, 2002.

[14] U.V. Marti, R. Messerli, and H. Bunke. Writer identification using text line based features. In Document Analysis and Recognition, 2001. Proceedings. Sixth International Conference on, pages 101-105. IEEE, 2001.

[15] L. O'Gorman, M.J. Sammon, and M. Seul. Practical Algorithms for Image Analysis. Cambridge University Press, 2008.

[16] L. Schomaker and M. Bulacu. Automatic writer identification using connected-component contours and edge-based features of uppercase western script. Pattern Analysis and Machine Intelligence, IEEE Transactions on, 26(6):787-798, 2004.

[17] I. Siddiqi and N. Vincent. A set of chain code based features for writer recognition. In Document Analysis and Recognition, 2009. ICDAR'09. 10th International Conference on, pages 981-985. IEEE, 2009.

[18] M. Sreeraj and M.I. Sumam. A survey on writer identification schemes. International Journal of Computer Applications, 26(2):23-33, 2011.

[19] S.N. Srihari, S.H. Cha, H. Arora, and S. Lee. Individuality of handwriting: a validation study. In Document Analysis and Recognition, 2001. Proceedings. Sixth International Conference on, pages 106-109. IEEE, 2001.

[20] L. Xu, X. Ding, L. Peng, and X. Li. An improved method based on weighted grid micro-structure feature for text-independent writer recognition. In Document Analysis and Recognition (ICDAR), 2011 International Conference on, pages 638-642. IEEE, 2011. 